\title{
Reconstrucción mamaria con colgajo musculocutáneo de recto abdominal con isla transversa de piel (TRAM): Experiencia de 12 años en Hospital El Pino
}

\author{
José Luis Román F. ${ }^{1}$, Michel Olivera I. ${ }^{1}$ y Gely Muñoz B. ${ }^{2}$
}

\section{Breast reconstruction with musculocutaneous flap of abdominal rectus with transverse skin island (TRAM): 12 years of experience at Hospital El Pino}

Objectives: To analyze the results of breast reconstruction with musculocutaneous flap of the rectus abdominis with transverse skin island (TRAM) in the Service of Surgery of El Pino Hospital. Materials and Methods: Retrospective study of patients undergoing mastectomy for breast cancer and subsequent reconstruction with pedicled TRAM flap in the last 12 years (2005-2017). Results: 12 mammary reconstructions were performed, 6 were with pedicled TRAM flap. The majority of patients received adjuvant treatment with radiotherapy ( 1 case), chemotherapy ( 1 case), chemotherapy + radiotherapy ( 2 cases). The time elapsed between the mastectomy and breast reconstruction was on average 2.5 years. There were 4 complications $(66.7 \%)$ that required reintervention: early postoperative hematoma, infection and flap fat necrosis, abdominal suture dehiscence, fat necrosis + TRAM flap fibrosis. The evaluation of the results was subjective, obtaining very good results in $66.7 \%$ of the cases. There were no complications in the donor site. Discussion: The pedicled TRAM flap is the most used autologous tissue in breast reconstruction. Its complication rate is $26 \%$, much lower than that obtained in our experience. The main complications are fibrosis and flap fat necrosis, flap loss, seroma and infection, with fat necrosis being the most frequent in our series. The use of TRAM flap gives greater satisfaction with appearance, size and sensation of the breast. Due to the results obtained, we believe that the pedicled TRAM flap is an excellent alternative for breast reconstruction in our hospital.

Key words: pedicled TRAM flap; mammary reconstructions; breast cancer; mastectomy.

\section{Resumen}

Objetivos: Analizar los resultados de reconstrucción mamaria con colgajo musculocutáneo de recto abdominal con isla transversa de piel (TRAM) en el Servicio de Cirugía del Hospital El Pino. Materiales y Métodos: Estudio retrospectivo de pacientes sometidas a mastectomía por cáncer de mama y posterior reconstrucción con colgajo TRAM pediculado en los últimos 12 años (2005-2017). Resultados: Se realizaron 12 reconstrucciones mamarias, 6 fueron con colgajo TRAM pediculado. La mayoría de las pacientes recibieron tratamiento adyuvante con radioterapia (1 caso), quimioterapia (1 caso), quimioterapia + radioterapia (2 casos). El tiempo transcurrido entre la mastectomía y reconstrucción mamaria fue en promedio de 2,5 años. Se produjeron 4 complicaciones $(66,7 \%)$ que necesitaron reintervención: hematoma posoperatorio precoz, infección y necrosis grasas de colgajo, dehiscencia de sutura abdominal, necrosis grasa + fibrosis de colgajo TRAM. La evaluación de los resultados fue subjetiva obteniéndose muy buenos resultados en el 66,7\% de los casos. No hubo complicaciones en sitio donante. Discusión: El colgajo TRAM pediculado es el tejido autólogo más utilizado en reconstrucción mamaria. Su tasa de complicación es de $26 \%$ muy por debajo a lo obtenido en nuestra experiencia. Las principales complicaciones son fibrosis y necrosis grasa de colgajo, pérdida de colgajo, seroma e infección siendo la necrosis grasa la más frecuente en nuestra serie. El uso de colgajo TRAM otorga mayor satisfacción con apariencia, tamaño y sensación del seno. Debido a los resultados obtenidos creemos que el colgajo TRAM pediculado es una excelente alternativa de reconstrucción mamaria en nuestro hospital.

Palabras clave: colgajo TRAM pediculado; reconstrucción mamaria; cáncer de mama; mastectomía.
Servicio de Cirugía Hospital El Pino.

2Facultad de Medicina Universidad Andrés Bello. Santiago, Chile.

Conflictos de interés: no hay

Recibido el 9 de enero y aceptado para publicación el 2 de mayo de 2018.

Correspondencia a: Dr. Michel Olivera I. mjrules23@hotmail.com 


\section{Introducción}

La reconstrucción mamaria, posmastectomía por cáncer de mama, ocupa un lugar importante del quehacer de la cirugía plástica. Según la literatura, el $40 \%$ de las pacientes con cáncer de mama es sometida a mastectomía y la mitad de ellas van a reconstrucción ${ }^{1,2}$.

El objetivo de la reconstrucción mamaria es lograr que la mujer recupere su contorno corporal previo y así superar el trance psicológico que supone la pérdida de un órgano tan íntimo para la vida personal, social y profesional. Dentro de las alternativas de reconstrucción mamaria tenemos los implantes protésicos, colgajo con tejido autólogo y combinaciones de ambos ${ }^{3}$.

La reconstrucción protésica es la técnica más frecuentemente utilizada en Chile y el mundo ${ }^{1,2}$. Sin embargo, el colgajo musculocutáneo de recto abdominal con isla transversa de piel TRAM se ha transformado en el gold standard en muchos países siendo el método reconstructivo autólogo más utilizado ${ }^{4,5}$. Según reportes de la Sociedad Americana de Cirujanos Plásticos, el año 2016 se realizaron 109.256 reconstrucciones mamarias de las cuales $5.190(4,8 \%)$ fueron realizadas con colgajo TRAM ${ }^{6}$.

Nuestro objetivo en este trabajo es dar a conocer la experiencia obtenida en la reconstrucción mamaria mediante el uso de colgajo TRAM pediculado en un hospital público chileno que no es centro de referencia.

\section{Materiales y Métodos}

Se realizó un estudio descriptivo y retrospectivo para lo cual se revisaron fichas clínicas utilizando como criterios de inclusión pacientes sometidas a mastectomía por cáncer de mama y posterior reconstrucción mamaria con colgajo TRAM operadas entre los años 2005 y 2017 en el Hospital El Pino. Se excluyeron pacientes con mastectomía por cáncer de mama y/o reconstrucción mamaria con colgajo TRAM realizadas en otros centros de salud con seguimiento posterior en nuestro hospital.

Dentro de las variables analizadas se consideró sexo, edad, tipo de cáncer de mama según resultado de biopsia, mama afectada, comorbilidades, hábito tabáquico, tipo de cirugía oncológica, tipo de tratamiento adyuvante previo a la reconstrucción, fecha de reconstrucción mamaria, lateralidad de colgajo TRAM, tiempo entre mastectomía y reconstrucción, complicaciones posoperatorias (dentro de los primeros 30 días posoperatorios), reintervenciones, manejo de complicaciones, tiempo de hospitalización y resultados los cuales se evaluaron de manera subjetiva por el propio cirujano plástico que realizó la reconstrucción.

Para el análisis de los datos obtenidos se utilizó principalmente estadística descriptiva, considerando los datos como variables cuantitativas expresadas en números absolutos y porcentajes.

En cuanto a los aspectos éticos para la realización del trabajo, se presentó una solicitud al comité de ética de nuestro hospital, posterior a su aprobación se solicitó el consentimiento informado a cada paciente para su participación en nuestra investigación.

\section{Resultados}

Entre los años 2005 y 2017 se realizaron 96 mastectomías totales y 12 reconstrucciones mamarias de las cuales $6(50 \%)$ fueron con colgajo TRAM pediculado en mujeres con edades entre 43 a 63 años (promedio 51,8 años). Los diagnósticos previos a la reconstrucción mamaria fueron: cáncer de mama ductal in situ recidivado (1 caso), cáncer ductal infiltrante ( 3 casos) y cáncer lobulillar infiltrante (2 casos). La mama afectada correspondió a derecha en 2 casos $(33,3 \%)$ e izquierda en $4(66,7 \%)$.

Dentro de las comorbilidades destacaban hipertensión arterial $(50 \%)$, resistencia a insulina $(16,7 \%)$, hipotiroidismo $(16,7 \%)$ y depresión $(16,7 \%)$, ninguna paciente fumaba (Tabla 1$)$.

La cirugía inicial por cáncer de mama fue: mastectomía total derecha (1 caso), mastectomía total izquierda (3 casos), mastectomía total derecha + reconstrucción inmediata con TRAM (1 caso) y mastectomía total izquierda + reconstrucción inmediata con TRAM (1 caso).

Como tratamiento adyuvante previo a reconstrucción mamaria se utilizó radioterapia (1 caso; $16,7 \%$ ), quimioterapia ( 1 caso; $16,7 \%$ ), quimioterapia + radioterapia ( 2 casos; $33,3 \%$ ) la cual se realizó en 1 caso de reconstrucción mamaria inmediata.

El colgajo TRAM pediculado fue ipsilateral en la mayoría de los casos $(4 ; 66,6 \%)$, de ellos 3 recibieron radioterapia previa a la reconstrucción, siendo la opción utilizada para los 2 casos de reconstrucción mamaria inmediata. El tiempo transcurrido entre la mastectomía y reconstrucción mamaria fue en promedio de 2,5 años.

Se produjeron 4 complicaciones $(66,6 \%)$, las cuales necesitaron reintervención: hematoma posoperatorio precoz (revisión y hemostasia), infección y necrosis grasa de colgajo (regularización de colgajo + injerto de grasa en segundo tiempo), dehiscencia 
de sutura abdominal (resutura) y necrosis grasa + fibrosis de colgajo TRAM (resección de necrosis y fibrosis + lipoinyección). El tiempo de hospitalización varió entre 2 y 10 días (promedio 6,2 días) (Tabla 2).

La evaluación de los resultados fue llevada a cabo de manera subjetiva por el mismo cirujano plástico basado en la satisfacción del mismo y de la paciente clasificándose como malo ( 0 caso), regular ( 1 caso; $16,7 \%$ ), bueno ( 1 caso, $16,7 \%$ ) y muy bueno (4 casos, 66,6\%). No hubo complicaciones en sitio donante.

\section{Discusión}

La reconstrucción mamaria ocupa un lugar importante en el quehacer de la cirugía plástica en parte, por la incidencia de cáncer de mama y por el impacto biopsicosocial sobre la mujer.

Actualmente existen múltiples alternativas re-

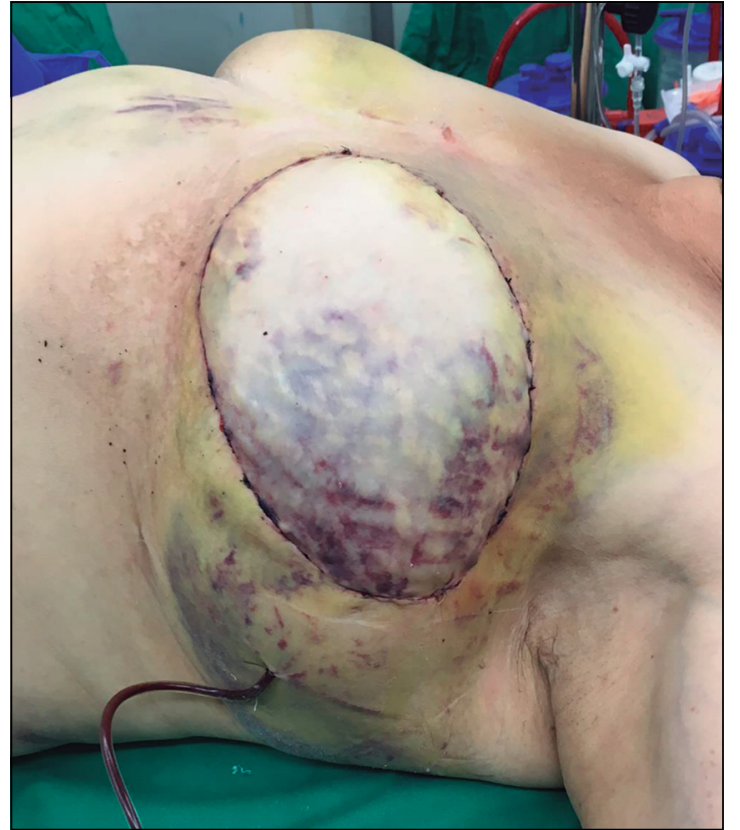

Figura 1. Complicación posoperatoria. Hematoma colgajo TRAM pediculado posoperatorio precoz.

Tabla 1. Características de Pacientes Sometidos a Reconstrucción Mamaria con Colgajo TRAM Pediculado en el Hospital El Pino

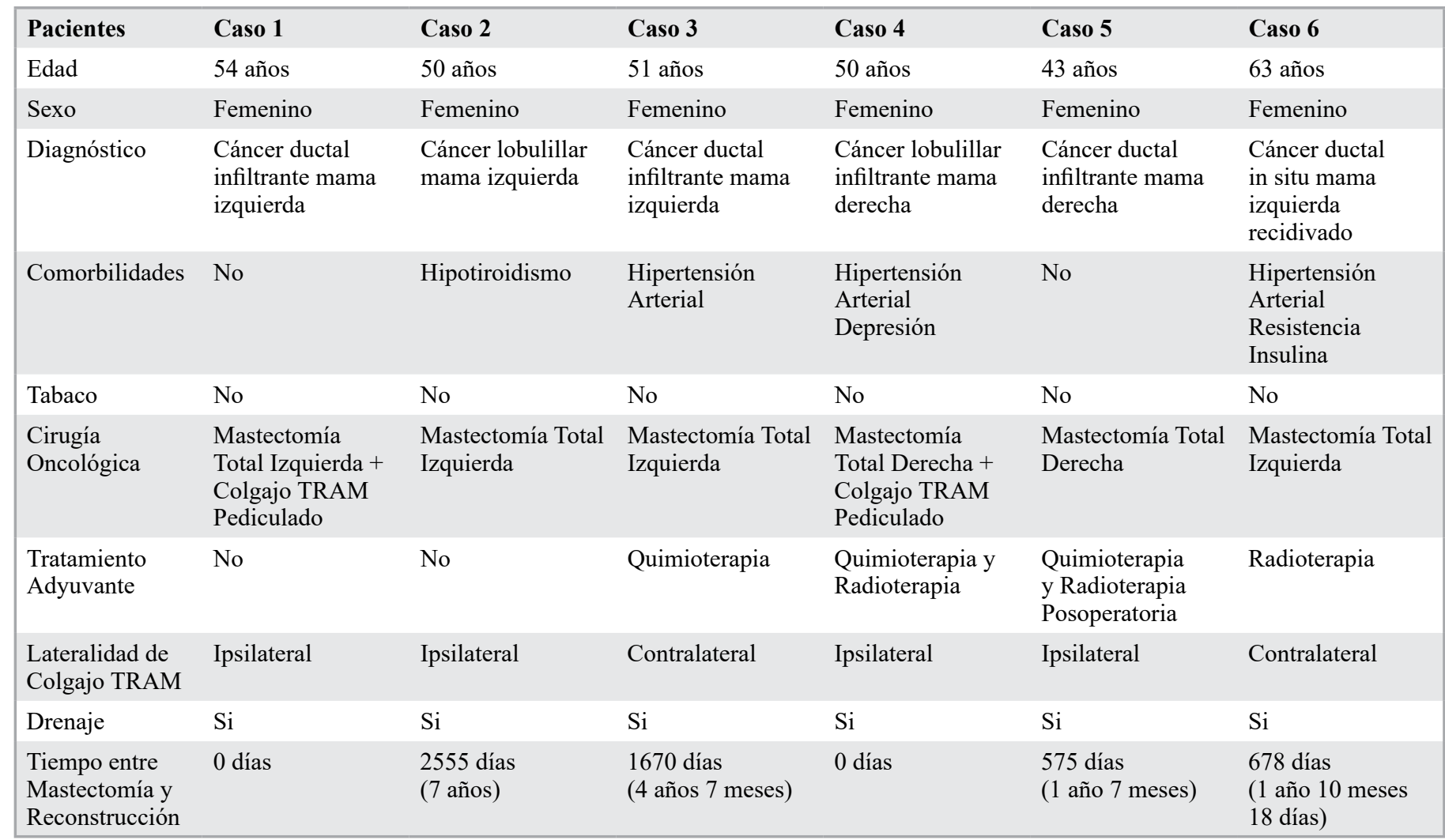


Tabla 2. Complicaciones posoperatorias y su tratamiento

\begin{tabular}{|c|c|c|c|c|c|c|}
\hline Pacientes & Caso 1 & Caso 2 & Caso 3 & Caso 4 & Caso 5 & Caso 6 \\
\hline Complicación & $\begin{array}{l}\text { Necrosis grasa } \\
\text { Fibrosis colgajo } \\
\text { TRAM }\end{array}$ & No & $\begin{array}{l}\text { Dehiscencia } \\
\text { sutura abdominal }\end{array}$ & $\begin{array}{l}\text { Infección y } \\
\text { necrosis grasa } \\
\text { Colgajo TRAM }\end{array}$ & No & $\begin{array}{l}\text { Hematoma } \\
\text { posoperatorio } \\
\text { colgajo TRAM }\end{array}$ \\
\hline Reintervención & $\mathrm{Si}$ & No & $\mathrm{Si}$ & $\mathrm{Si}$ & No & $\mathrm{Si}$ \\
\hline $\begin{array}{l}\text { Manejo de } \\
\text { complicación }\end{array}$ & $\begin{array}{l}\text { Resección de } \\
\text { Necrosis grasa y } \\
\text { fibrosis colgajo }+ \\
\text { lipoinyección }\end{array}$ & No & Resutura & $\begin{array}{l}\text { Regularización de } \\
\text { colgajo + injerto } \\
\text { de grasa }\end{array}$ & No & $\begin{array}{l}\text { Drenaje de } \\
\text { hematoma }+ \\
\text { hemostasia }\end{array}$ \\
\hline $\begin{array}{l}\text { Tiempo de } \\
\text { hospitalización }\end{array}$ & 10 días & 5 días & 7 días & 7 días & 2 días & 6 días \\
\hline Resultado & Conforme & Conforme & Muy bien & Muy bien & Muy bien & Muy bien \\
\hline
\end{tabular}

constructivas para ofrecer, siendo la forma más común el uso de implantes, sin embargo, esta técnica no está exenta de complicaciones y requiere de múltiples revisiones a lo largo del tiempo ${ }^{1}$. Por tal motivo se han buscado otras alternativas de reconstrucción basadas principalmente en el uso de tejidos autólogos destacando últimamente las técnicas microquirúrgicas.

Existen varias opciones de reconstrucción con tejido autólogo siendo la más comúnmente utilizada el colgajo musculocutáneo de recto abdominal con isla transversal de piel infraumbilical (TRAM) que se caracteriza por sus excelentes resultados estables en el tiempo $2,3,7$.

En nuestra experiencia, la elección del colgajo TRAM pediculado para reconstrucción mamaria se basa en la falta de recursos para adquirir los implantes, el no ser un centro de referencia para reconstrucción mamaria, la necesidad de radioterapia y/o quimioterapia posmastectomía y contar con un único cirujano plástico-reconstructivo.

$\mathrm{Si}$ bien nuestra serie de casos es pequeña, los resultados obtenidos se asemejan a los reportados en la literatura con series mucho más grandes y centros con mayor experiencia. Es así que la edad de nuestras pacientes fluctúa entre los 43 y 63 años (promedio de edad 51,8 años, levemente superior al promedio reportado en otra experiencia nacional previa $^{7}$, pero similar a series extranjeras). Al igual que en otros estudios el carcinoma ductal infiltrante fue el tipo histológico más frecuentemente intervenido $^{7}$.

Las principales comorbilidades que presentan las pacientes sometidas a reconstrucción mamaria con tejido autólogo y que influyen en los resultados obtenidos son la obesidad, diabetes mellitus, hipertensión arterial y tabaquismo. Destacamos el hecho que ninguna de nuestras pacientes fumaba, solo presentaban hipertensión arterial $(50 \%$ de los casos) y sobrepeso. Importante mencionar la necesidad de radioterapia, quimioterapia o radioterapia + quimioterapia posoperatoria $(66,6 \%$ de los casos) que es mayor a lo reportado en otras series (entre 5 a $12 \%$ ) hecho que podría explicar la alta incidencia de complicaciones en nuestra experiencia.

Respecto al momento de la reconstrucción mamaria esta puede ser inmediata (al momento de la mastectomía), temprana (dentro de 1 año) o tardía (más de 1 año $)^{3}$. De acuerdo con las guías de práctica clínica para cáncer de mama en Chile, las pacientes que requieren mastectomía sin radioterapia adyuvante son candidatas a reconstrucción mamaria inmediata la cual resulta segura y efectiva. En el caso de requerir radioterapia adyuvante se recomienda la reconstrucción diferida ${ }^{3}$. Por tal razón más de la mitad de nuestras pacientes se reconstruyeron en forma tardía (2,5 años promedio).

En cuanto a las complicaciones para la reconstrucción mamaria con colgajo TRAM pediculado, estas muestran una tasa cercana al $26 \%{ }^{8}$. Varios son los aspectos que influyen en las complicaciones que presenta el colgajo TRAM pediculado en reconstrucción mamaria, siendo los más importantes: radioterapia, quimioterapia, tabaquismo, obesidad, hipertensión arterial y diabetes mellitus9.

La radioterapia contribuye a peor resultado estético, mayor tasa de seroma, fibrosis y necrosis grasa del colgajo ${ }^{3,10,11}$, la quimioterapia se asocia a complicaciones precoces como alteración en cicatrización de heridas y complicaciones tardías como necrosis grasa ${ }^{10}$; tabaquismo afecta la cicatrización y el flujo sanguíneo incrementando el riesgo de infec- 
ción de herida, necrosis grasa, necrosis de colgajo y hernia abdominal ${ }^{10,11}$, obesidad se asocia con mayor dificultad técnica, tiempo operatorio prolongado, mayor costo en hospitalización y mayor riesgo de complicaciones como pérdida de colgajo, necrosis de colgajo y hematoma ${ }^{10,12}$, hipertensión arterial se asocia con complicaciones menores y mayores, diabetes mellitus aumenta la tasa de complicaciones por predisposición a la infección y trastorno de la micro y macrocirculación ${ }^{10}$.

Lo anterior se ve reflejado en el tipo de complicaciones que obtuvimos: necrosis grasa + fibrosis de colgajo TRAM, infección + necrosis grasa de colgajo TRAM, dehiscencia de sutura abdominal y hematoma de colgajo TRAM; siendo la más frecuente la necrosis grasa del colgajo tal como se reporta en otras series ${ }^{8}$. Del análisis de dichas complicaciones la mayoría se presenta en pacientes sometidas a radioterapia y/o quimioterapia e hipertensión arterial no guardando clara relación con la lateralidad del colgajo (50\% de complicaciones en colgajo ipsilateral y $50 \%$ restante en colgajo contralateral). Debemos destacar la alta incidencia de complicaciones en nuestra experiencia con el uso de colgajo TRAM pediculado $(66,6 \%)$ que supera ampliamente lo reportado en la literatura; quizás esto se deba al pequeño tamaño de la muestra, mala selección de pacientes, mayor requerimiento de radioterapia/ quimioterapia o a la propia falta de experiencia.

A pesar de las complicaciones, los resultados obtenidos con el uso de colgajo TRAM pediculado en reconstrucción mamaria son bastante satisfactorios, en parte porque el tejido autólogo abdominal proporciona buena calidad y cantidad de tejido necesario para la reconstrucción ${ }^{13}$. La medición de dichos resultados en nuestra experiencia y otras locales se ha llevado a cabo de manera subjetiva basado principalmente en criterios del propio cirujano plástico, obteniendo un grado de satisfacción muy bueno en más de $2 / 3$ de los $\operatorname{casos}^{8}$. Sin embargo, a nivel internacional, existen herramientas como el BREAST-Q que permiten una medición estandarizada de los resultados estéticos y con ello una interpretación más confiable de los mismos ${ }^{8,14,15}$. Es así como mediante el uso de BREAST- $Q$ se ha podido concluir, en un estudio de Schwitzer et al, que con el uso de colgajo TRAM pediculado en reconstrucción mamaria las pacientes presentan mayor satisfacción con la apariencia, tamaño y sensación del seno dentro de los primeros 3 años posreconstrucción comparado con el uso de colgajo TRAM libre ${ }^{12}$.

Finalmente, es importante señalar que nuestro trabajo cuenta con ciertas limitantes dadas por el pequeño tamaño de la muestra que puede contribuir al sesgo de información y resultados, registro incompleto de datos en algunas fichas médicas, poca experiencia en reconstrucción mamaria (que se relaciona directamente con los resultados) y evaluación subjetiva de resultados obtenidos que puede constituir un sesgo.

\section{Responsabilidades éticas}

Protección de personas y animales. Los autores declaran que para esta investigación no se han realizado experimentos en seres humanos ni en animales.

Confidencialidad de los datos. Los autores declaran que en este artículo no aparecen datos de pacientes.

Derecho a la privacidad y consentimiento informado. Los autores declaran que en este artículo no aparecen datos de pacientes.

\section{Referencias}

1. Sepúlveda S. Reconstrucción Mamaria. Rev Med Clin Condes 2016;27:65-75.

2. Marré D, Gantz J, Villalón J, Roco H, Reconstrucción Mamaria: Estado Actual del Tema. Rev Chil Cir. 2016;68:186-93.

3. Reconstrucción Mamaria. En: Subsecretaria de Salud Pública, División de Prevención y Control de Enfermedades, Departamento Manejo Integral del Cáncer y otros tumores, Ministerio de Salud de Chile, Guías Clínicas AUGE Cáncer de Mama. Santiago: Minsal 2015;97-105.

4. Caterson SA, Carty MJ, Helliwell LA, Hergrueter CA, Pribaz JJ, Sinha I. Evolving options for breast reconstruction,
Curr Probl Surg. 2015;52:192-224.

5. López JL, Gutiérrez JE, Dávila R, Poucel F, Barrera M. Complicaciones en el uso del Colgajo TRAM pediculado para reconstrucción mamaria por cáncer. Cir Plast Iberolatinoam. 2013;39:381-91.

6. American Society of Plastic Surgery. 2016 Plastic Surgery Statistics Report. http:// www.plasticsurgery. org/Documents/ news-resources/statistics/2016-PlasticSurgery-Statistics/full-plastic-surgerystatistics-report. pdf.

7. Nahabedian M, Patel K. Autologous Flap Breast Reconstruction: Surgical Algorithm and Patient Selection. J Surg Oncol. 2016;113:865-74.

8. Ibáñez M, Ibáñez G, Pereira N, Mandiola
C, Andino R. Reconstrucción Mamaria con Colgajo TRAM Pediculado. Rev Chil Cir. 2012;64:40-5.

9. Wang XL, Liu LB, Song FM, Wang QY. Meta-analysis of the safety and factors contributing to complications of MSTRAM, DIEP, and SIEA flaps for breast reconstruction. Aesthetic Plast Surg. 2014;38:681-91.

10. Voineskos SH, Frank SG, Cordeiro PG. Breast reconstruction following conservative mastectomies: predictors of complications and outcomes, Gland Surg. 2015;4:484-96.

11. Selber JC, Kurichi JE, Vega SJ, Sonnad SS, Serletti JM. Risk factor and complications in free TRAM flap 


\section{ARTÍCULO ORIGINAL}

breast reconstruction, Ann Plast Surg. 2006;56:492-7.

12. Moran SL, Serletti JM. Outcome comparison between free and pedicled TRAM flap breast reconstruction in the obese patient, Plast Reconstr Surg. 2001;108:1954-60.
13. Traverso J. Reconstrucción mamaria con TRAM pediculado, Revista Argentina de Cirugía Plástica 2015;3:94-101.

14. Schwitzer JA, Miller HC, Pusic AL, Matros E, Mehrara BJ, McCarthy CM, et al. Satisfaction following Unilateral Breast Reconstruction: A Comparison of Pedicled
TRAM and Free Abdominal Flaps. Plast Reconstr Surg Glob Open 2015;3:1-8.

15. Guyomard V, leinster S, Wilkinson M. Systematic review of studies of patients'satisfaction with breast reconstruction after mastectomy. Breast 2007; 16:547-67. 